Review

\title{
Health risks associated with genetic alterations in internal clock system by external factors
}

\author{
Suliman Khan ${ }^{1,2,3}$, Ghulam Nabi1,3, Lunguang $\mathrm{Yao}^{2 \bowtie}$, Rabeea Siddique ${ }^{4}$, Wasim Sajjad ${ }^{3,5}$, Sunjeet Kumar ${ }^{1}$, \\ Pengfei Duan ${ }^{2}$, Hongwei Hou ${ }^{1 凶}$
}

1. The Key Laboratory of Aquatic Biodiversity and Conservation of Chinese Academy of Sciences, Institute of Hydrobiology, Chinese Academy of Sciences, Wuhan, Hubei, 430072, P.R China.

2. Henan Key Laboratory of Ecological Security for Water Source Region of Mid-line of South-to-North Diversion Project, Collaborative Innovation Center of Water Security for Water Source Region of Mid-line of South-to-North Diversion Project of Henan Province, Nanyang Normal University.

3. University of Chinese Academy of Sciences, Beijing 100049.

4. Advanced Biomaterials and Tissue Engineering Center and Department of Biomedical Engineering, Huazhong University of Science and Technology, Wuhan 430074, China.

5. Key Laboratory of Petroleum Resources, Gansu Province / Key Laboratory of Petroleum Resources Research, Institute of Geology and geophysics, Chinese Academy of Sciences, Lanzhou, 730000, China

$\square$ Corresponding authors: Prof. Dr. Hongwei Hou, Institute of Hydrobiology, University of Chinese academy of Sciences. Email: houhw@ihb.ac.cn and Prof. Dr. Lunguang Yao, Nanyang Normal University, Nanyang, Henan, China. Email: lunguangyao@163.com

(c) Ivyspring International Publisher. This is an open access article distributed under the terms of the Creative Commons Attribution (CC BY-NC) license (https://creativecommons.org/licenses/by-nc/4.0/). See http://ivyspring.com/terms for full terms and conditions.

Received: 2017.11.08; Accepted: 2018.03.28; Published: 2018.05.21

\begin{abstract}
The circadian system maintains the main aspects of physiological and behavioral processes. Both circadian rhythm and sleep impact an organism's health through interaction with environmental factors. Variations in external factors influence the functionality of clock genes and disrupt 24-hour rhythmic cycle. The disrupted circadian rhythm and disregulated sleep affect an organism's health, thereby causing several disorders including cancer, depression and cardiac disorders. Considering the role of clock genes and environmental factors, extensive investigation is required focusing on pathways involved in development of life-threatening disorders. This review identifies the major risks and associated factors related with disruption in circadian system and sleep.
\end{abstract}

Key words: Circadian system, sleep, health risks, clock genes

\section{Introduction}

Specific phases of the day-night cycle, are linked to an internal biological clock that influences behavioral and physiological responses through synchronization of internal processes. The circadian system (comprising a network of tissue clocks) is coordinated in the suprachiasmatic nucleus (SCN) of the anterior hypothalamus by central pacemaker. Rhythmic transcriptional pathways are driven by molecular clock machinery in a tissue-specific manner. Proteins encoded by clock genes generate circadian rhythms in the SCN [1]. A circadian pacemaker is directly synchronized by light to the solar cycle (24-h). This synchronization (entrainment) process is an interplay between period of intrinsic pacemaker and daily light perception. The circadian pacemaker in SCN synchronizes sleep-wake cycle, hormone secretion, skin temperature and heart rate [2].

Circadian clock regulates early and late chronotypes through synchronization of the internal body time with the external cycle of light and dark. Light is associated with the wake, and dark with sleep period. However, self-selected sleep and wake timing differences are possible that are linked to specific chronotypes. Opposite to the late chronotypes, early chronotypes wake up and fall asleep early in the morning and evening respectively [3]. Irregular sleeping periods and altered light-dark cycles in shift-work and long distance travel are associated with altered environment cues, which further influence daily rhythms via central and peripheral clocks [4]. 
Adverse impacts of circadian dysrhythmia on body and mind are linked with lifestyle or routines such as frequently flying, working in repeatedly changing shifts and exposure to irregular light-dark conditions. Working around the clock and jetting around the globe are becoming necessary in our globalized world in some high-profile services. Crossing multiple time zones results in "jet lag," a circadian rhythm disorder, that disturbs spatial cognition and hippocampal neurogenesis. Jet lag immediately causes fatigue and indigestion while chronic exposure to jet lag has strong impacts on spatial cognition and temporal lobe [5]. Externally induced disruption in natural sleep-wake cycles [6] and circadian rhythms is associated with neuronal dysfunction $[7,8]$, increased risk for psychiatric, cardiovascular and metabolic diseases [3], and affects bone health [9].

Long-term exposure to irregular dark-light cycles increases the risk of sleep disorders, metabolic disorders, mental abnormalities, depressive disorders, and hormonal abnormalities, that further develop cancer, gastrointestinal, and reproductive disorders [10]. Trans meridian travel may develop general malaise, headaches, insomnia, daytime sleepiness, impaired cognitive or physical performance and gastrointestinal disturbances. Impacts of shift-work on sleep duration quality can disrupt the physiological rhythms via desynchronization between hormonal rhythms, metabolic function and sleep cycle [4]. Physiological clock alterations [11] increase the risk of peptic ulcer and diabetes [12-14].

Exposure to irregular light-dark cycles (shift-work) and jet lag experiences are unavoidable in order to fulfill the fast-growing needs. Environmental and other external factors impact circadian rhythms and sleep regulations. These problems need proper investigation and development of preventive or curing techniques. Genetic mechanisms associated with severe diseases and circadian system and sleep regulation disruptions promoted by shift work, jet lag and other environmental factors, need to be investigated.

\section{Circadian System}

Circadian system is regulated by clock genes with the effects of oscillators, endocrine, and neural signals. However, circadian rhythm is maintained by a circadian clock through appropriate physiological activities [15]. Circadian clock organization at cellular level takes place through brain and muscle ARNT like protein 1 (BMAL1) and circadian locomotor output cycles kaput (CLOCK) (Figure, 1). Two different loops are formed for the complete organization and activation of the circadian clock [16]. In the first loop, CLOCK and BMAL1, positive clock arm comprising elements, heterodimerize in the cytoplasm and enter the nucleus to regulate the expression of negative core clock arm comprising elements which are; PER1, PER2, and PER3 and CRY1, CRY2. Various Per and Cry protein combination in cytoplasm interact with each other and enter the nucleus to inactivate BMAL1/CLOCK complexes. The expression of secondary feedback loop elements "REV-ERB $a$ and $\mathrm{ROR} a$ " is driven by CLOCK/BMAL1 that regulates Bmal1's rhythmic expression [16]. RORa and REV-ERBa activate and represses BMAL1 expression respectively.

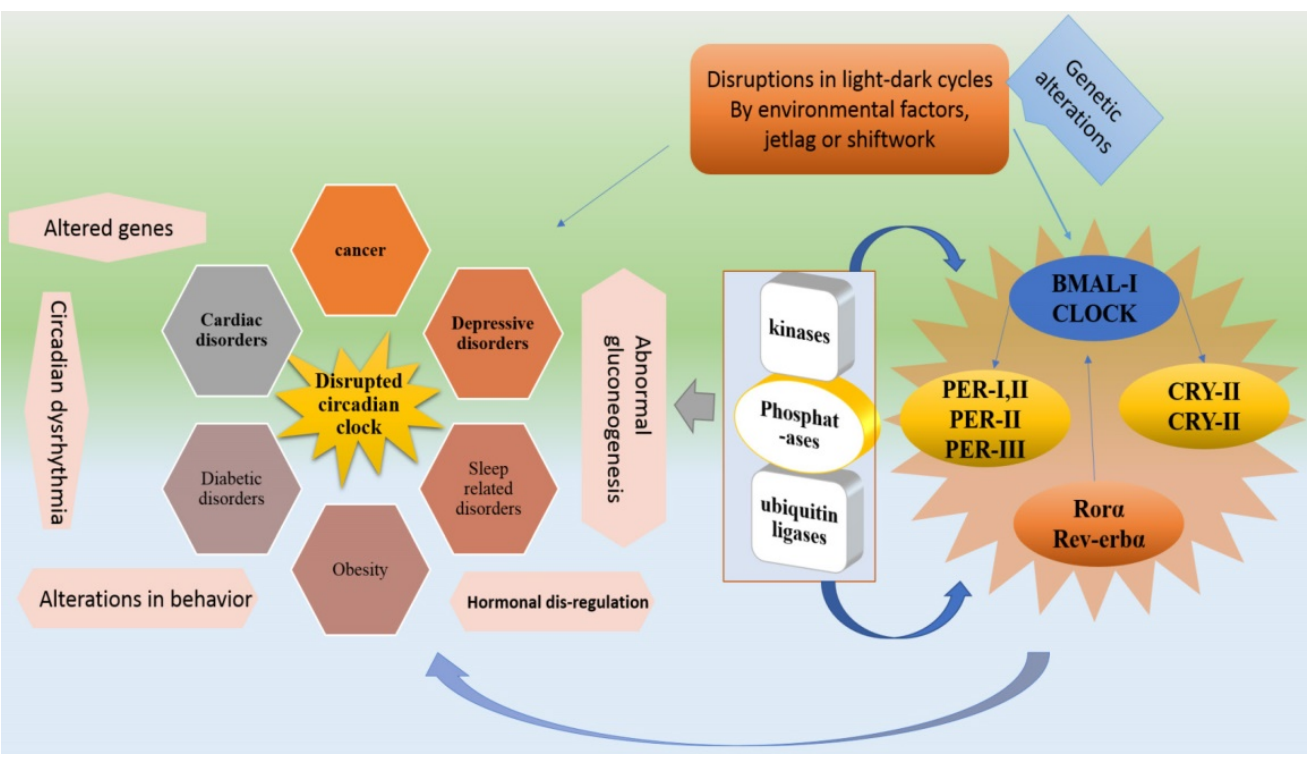

Figure 1, shows the genetic process of circadian system disruption by external factors and the resulted disorders. External influences such as shiftwork and jetlag dysregulate the light-dark cycles which disrupt Bmall, Clock, Cry and Per genes by causing alterations in RORa, Rev-erba, kinases, phosphatases and ubiquitin ligases. These disruptions impose their influences on physiological functions. In addition to cancer and obesity external factors mediated disruptions cause abnormal gluconeogenesis, hormonal dysregulation, cardiac, diabetic, sleep and depressive disorders. 
In case of activation, BMAL1 protein forms heterodimers with CLOCK that enters the nucleus to initiate the next cycle of gene activation of both loops $[17,18]$. Hence, the BMAL1's circadian oscillation is regulated by RORs and REV-ERBs (positively and negatively). The auto regulatory feedback loops constitute a circadian molecular clock after completing a cycle in a 24-hour time-period. This timing of molecular clock is regulated by post-translational modification proteins, including kinases, phosphateases, and ubiquitin ligases that affect the heterodimerization and degradation of PER and CRY [19].

Altered light-dark cycles or environmental factors can dysregulate clock either inactivating or overexpressing genes of core Clock genes. Core Clock genes and related factors if mutated, can lead to several abnormalities. For instance, Bmal1 and Clock mutations can cause abnormal gluconeogenesis, behavioral sensitization to psychostimulants and lipogenesis, and altered sleep pattern. Per1 and Per2 can lead to developing cancer. Cry1, Cry2 and Rora mutations can develop altered sleep pattern and cerebellar ataxia respectively. Rorb dysfunctionality is related to locomotor difficulties and retinal degeneration [19]. Moreover, Some important metabolic process controlling genes are present in rhythmic transcriptome which is an indication of the fact that alteration in circadian rhythm causes metabolic problems [15,20-22]. For instance, Clock, Bmal and Per-2 are associated with obesity $[23,24]$.

Circadian rhythms are synchronized with the day time work and nighttime sleep, require a phase adjustment (as occur with exposure to bright light at night, experiencing jet lag or shift work) by central and peripheral oscillators. Because Per1 and Per2 shift faster than Cry1, the phase adjustment in certain cases causes internal desynchronization. Surprisingly, Per1 and Per2 behave differently during phase shifts (jet lag or shiftwork) $[6,25]$ that may ultimately cause the disruption in the circadian clock. Along with sleep regulation, circadian rhythms also direct normal physiological processes, including hormone secretion, body temperature, feeding behavior, cell-cycle progression and drug, glucose and xenobiotic metabolism $[10,22]$.

\section{Disregulated Circadian Rhythm Disrupts Sleep}

Sleep is a critical component of human health. This sleep health mainly depends on factors determining sleep quantity and quality. Several sleep and circadian rhythms related physiological functions including the sleep/wake cycle and metabolism are maintained by the SCN of the hypothalamus [26]. Insufficient and poor sleep is associated with increased risk of metabolic disorders and exacerbating metabolic outcomes [27]. Light exposure influences human physiology including sleep modulation. Its impacts on circadian physiology cause circadian misalignment that further alters sleeping energy expenditure and affects substrate oxidation. The alterations in $24 \mathrm{~h}$ energy expenditure increase the risk of obesity [28].

Environmental stimuli (light at night) mediated sleep disruption is considered one of the potential risk factors for cardiovascular disease, dysregulation of immune function, stress, memory deficits and depression. Being the main environmental signal for circadian clock synchronization, light exposure in the early morning and late evening is associated with shifting of circadian phase [29]. Poor sleep develops endocrinal, psychiatric, cardiovascular and metabolic abnormalities. Its elevated affects are coronary artery calcifications, atherogenic lipid profiles, atherosclerosis, obesity, type 2 diabetes, and hypertension (Figure 2). Nocturnal environmental noise affects sleep architecture and quality in a similar way to that observed in endogenous sleep disorders. Being an important modulator sleep modulates cardiovascular function, hormonal release and glucose regulation. Both sleep restriction, and poor quality, reduces glucose tolerance and insulin sensitivity [30].

Several aspects of human behavior and physiology are dominated by 24-h circadian rhythms including vigilance and sleep-wake cycle. An internal pacemaker generates rhythms spontaneously based on daily retinal light exposure. Such synchronization processes pose problems in the context of jet lag or working night shifts [31]. Sleep is controlled genetically with the influence of environmental factors [32]. Sleep and circadian rhythms influence physiologic processes which are critical to brain health [33,34]. Sleep consists of two repeated cyclic patterns "non-rapid eye movement (NREM) and rapid eye movement" and regulated by cyclic guanosine monophosphate (cGMP) kinase, regulatory subunit of Shaker, Sleepless (sss) gene and CLK and CYC proteins are the key players in sleep regulation [10].

Externally influencing factors may alter genetic expressions to develop sleep disorders. It can affect an SNP marker, MEIS1 locus and Neuronal nitric oxide synthase (NOS1) [10,35-37]. These factors can disrupt the normal functions of Per2, casein kinase I $\delta$ and PRNP genes to develop Familial advanced sleep phase syndrome (FASPS) and fatal familial insomnia (FFI) respectively [10]. They can also affect daytime activity increasing glucocorticoids and NSAIDs [38], Protein NF-kB (up-regulated during sleepdeprivation) [32], TAK1 (TGF-b-activated kinase) and Sik3 (control sleep behaviors), Nalcn gene (involved 
in REMS) [39]. Furthermore, expression of Sur2 gene (play role in energy metabolism) [40], daily patterns of human physiology, irregular energy patterns expenditure, hormones and lipids involved in energy metabolism [41,42] can be affected (Figure, 2).

\section{Major Health Problems}

Genetic variation, environmental factors and jetlag increase the risks of a wide range of health abnormalities through altering circadian rhythms [1]. Desynchrony induced by external/environmental factors is linked with high risk of serious health issues, including depressive disorders, cardiovascular disorders, diabetes and cancer $[1,43]$. Social jet lag is associated with depressive and metabolic syndromes that further enhance the consumption of nicotine, caffeine and alcohol [3] while inappropriately timed sleep is associated with endocrine, metabolic, cardiovascular, and neurological disorders [9]. Incompatibility between circadian rhythms and working periods causes sleep deprivation [44] that further causes fatigue, vigilance and attention impairments [45] (Figure 3).

\section{Delayed/advanced phase sleep syndrome}

Forcing late chronotypes to wake up early or early chronotypes to wake to up late irrespective to their biological wake-up time will disrupt the circadian clock system. This condition, if extenuated may lead to delayed or advanced sleep phase syndrome. Advanced sleep phase syndrome (ASPS) is inherited in an autosomal dominant mode where mutations in S662G and Csnk1d increase the risk [22]. In case of delayed sleep phase syndrome (DSPS) T3111C polymorphism in the 3'UTR of Clock, SNP association in 5'UTR of Per2 and mutation in Cskn1e gene, play a key role $[22,46,47]$ where Per3 gene performs dominantly [48].

Mutation in hPER2 is linked both with Advanced and Delayed Sleep Phase Syndromes. Alterations into casein kinase $\mathrm{I} \varepsilon(\mathrm{CKI} \varepsilon)$ and polymorphisms in $3^{\prime}$ flanking region clock homolog $(3111 \mathrm{~T} / \mathrm{C})$ are associated with evening-ness and morning-ness [47].

\section{Familial Advanced sleep phase syndrome}

Familial advanced sleep-phase syndrome (FASPS) is a prototypical sleep disorder where the sleep and wake times are approximately three hours earlier than normal routines. In specific circumstances where healthy people tend to delay their wake-sleep times, FASPS patients profoundly advance their wake-sleep time [49]. Development of FASPS is linked with Per1-3 genes where hPER2, Ser-662 plays an important role [50]. Alteration in kinase activities by
CK11 and CK1d mutation causes shorter circadian periods [19]. These genetic modifications indicate that FASPS can highly be induced by dysregulation of the circadian clock system and sleep.

\section{Bone health}

Unhealthy and weak bones increase the risks of injuries to the main organs including the brain and heart. Not only food, lifestyle and routine affect the bones, but also the disruptions in sleep and circadian rhythmicity have significant impacts on bone health. Daily rhythm in bone turnover markers and the clock genes existence in bone cells are indications for the circadian system and sleep role in bone health maintenance. It was observed in an experiment that clock gene knockout models resulted in altered skeletal phenotypes, and repeated sleep restriction arrests resulted in bone remodeling in rats [9].

\section{Obesity and Diabetes}

Environmental/external factors mediated disruptions in sleep and circadian system alter the hormonal release pattern that ultimately reduces leptin level and induces diabetes. People facing irregular light exposures, are at higher risk of type II diabetes [42,52]. Sleep timing is regulated by circadian machinery and clock-controlled genes ClockD19, Bmal1, Per1, Rev-erba and Dbp. Altered expression of these genes by irregular light and dark cycles, affect carbohydrate regulators such as insulin receptor substrate 2 (Irs2) and glucose transporter 2 (Slc2a2) [15]. An altered expression of these genes impact carbohydrates, and lipid utilization in the liver. Disturbance of this circadian control or environmental / behavioral cycle results in disturbed glucose control and increased type 2 diabetes risk [15].

Diabetes has reached epidemic proportions and the cases of type 2 diabetes are increasing uncontrollably. The involvement of approximately $20 \%$ of the working population in shift-work even increases the risks associated with obesity and diabetes [52]. Insufficient sleep reduces insulin sensitivity and leads to an increased risk of diabetes [53].

Sleep disruption reduces glucose tolerance while inducing insulin resistance which can ultimately lead to obesity and diabetes. The glucose impairment, in this case, is carried by suppressing glycolysis and enhancing gluconeogenesis [54].

\section{Sleep sickness}

Sleep sickness is basically a tropical disease which is caused by Trypanosoma brucei. It is characterized by sleepiness at day time while insomnia at night. The normal habitats of Trypanosoma brucei are bloodstream and interstitial 
spaces of skin and adipose tissue. It can invade the brain and cause death if left untreated. This infection advances the phase through shortening of the circadian period [26]. Environmental or external inducers increases the risk of sleep sickness as well. Sleep pattern disruption via circadian rhythm dysregulation is the primary factor that develop this disorder.

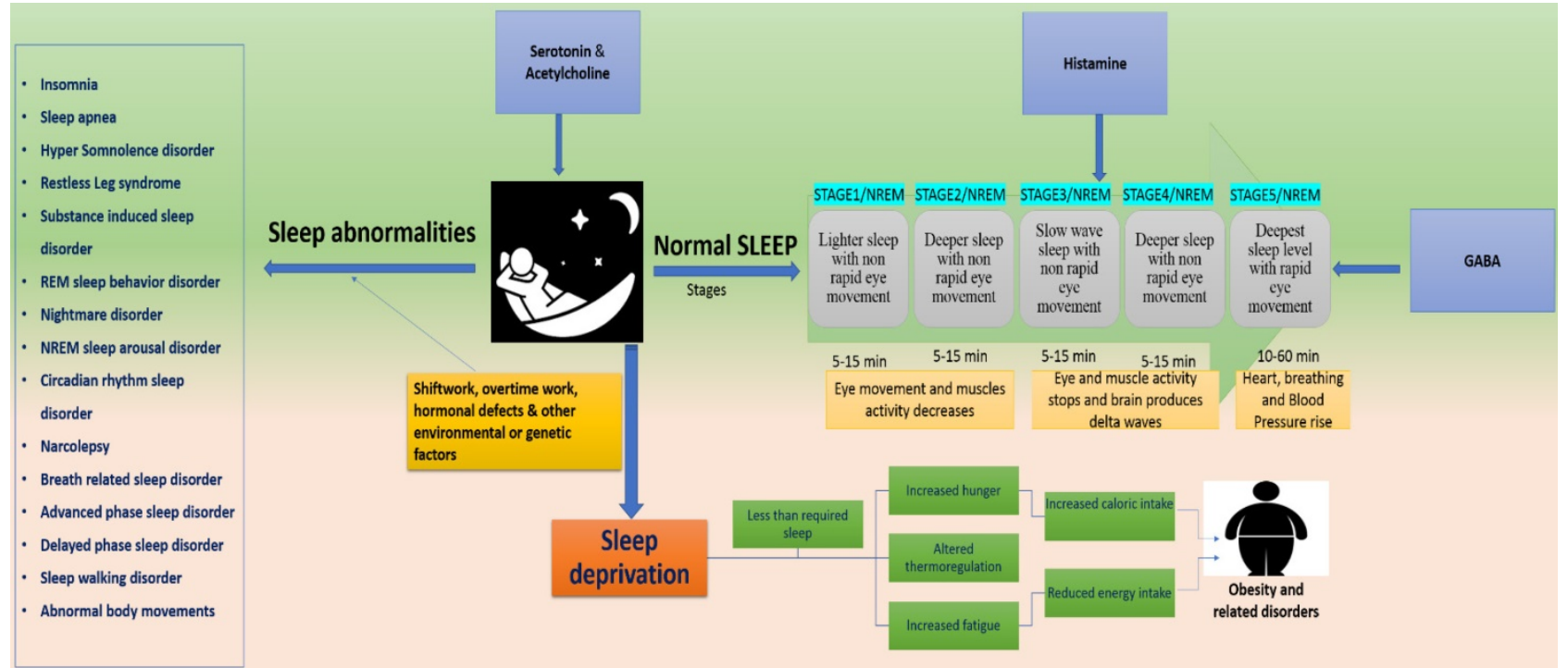

Figure 2, represents the disorders associated with disrupted sleep. Dysregulation at any stage of the 5 sleep stages, develop several health risking disorders. Serotonin, acetylcholine, GABA and histamine paly key role in sleep maintenance and their dysregulation can lead to sleep abnormalities or sleep deprivation. Sleep disruption is majorly associated with external influences such as shiftwork, jetlag and overtime work. These factors develop several disorders such as insomnia, sleep apnea, REM and NREM sleep disorders and delayed and advanced phase sleep syndromes. They can also cause sleep deprivation thereby causing obesity.

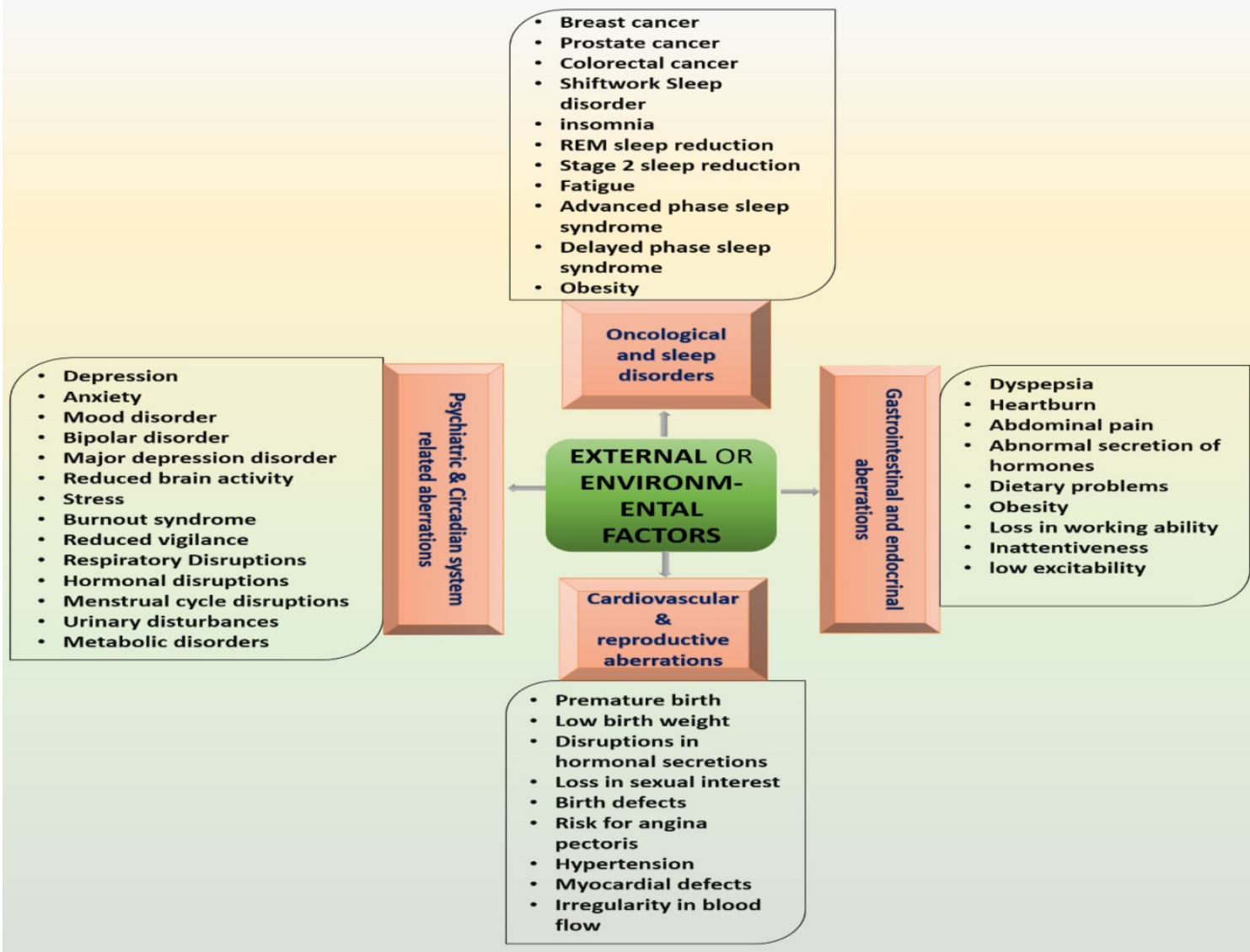

Figure 3, represents the major disorders that can be caused by external factors. These disorders are developed by the imposed effects of environmental influences on circadian and sleep system. 


\section{Cardiovascular disorders}

Physiological and pathological functions, including heart rate, blood pressure, and endothelial function, of cardiovascular organs are related to circadian rhythms. Circadian system, if disturbed, will develop atrial arrhythmia, acute coronary syndrome and subarachnoid hemorrhage as the peripheral clock, tissues with rhythmic expressions are present in the cardiovascular system. Being tissue or cell type specific peripheral clock in cardiovascular system decides the fate of different functions, and its loss or dysfunctionality can develop cardiovascular disorders. Per2 mutation reduces the nitric oxide level, which causes impairment in endothelium-dependent vasodilatory responses. PAR transcription factors (Dbp, Hlf) and Anp are mainly related to hypertrophy [55]. Long-term exposure to non 24 hours light-dark (LD) cycles causes physiological disturbance and depression of cardiac function [1].

\section{Depressive disorders}

Circadian system disruption as occurs in jetlag and shift-work has a strong impact on melatonin release, thereby increasing the risks of depressive disorders [56]. Specifically, late night lights exposure dysregulates the production of norepinephrine, melatonin and serotonin [57].

\section{Shiftwork sleep disorder}

Excessive sleepiness, insomnia, and reduction in alertness and performance are the major symptoms of the shift-work sleep disorder $[11,41,58,59]$. Among the shift-workers, $9 \%$ develop severe symptoms while $35 \%$ develop mild symptoms of shiftwork sleep disorder which can cause hypertension [59]. The development of shift-work sleep disorder is directly linked to the working time period and age [14]. Shift-work sleep disorder increases the risks of depression, ulcers and sleepiness-related accidents [60]. The association of 3111C/C with delayed shift of sleep [47] and melatonin with circadian clock shift [61] indicates the involvement of 3111C and melatonin in shift-work sleep disorder.

\section{Circadian rhythm sleep disorder}

Sleep-wake pattern variations increase the risk of circadian rhythm sleep disorder. Neuroendocrine physiology, glucose level and insulin sensitivity are mainly affected by circadian rhythm dysregulation $[42,51,62,63]$ which indicates that they can also get affected with circadian rhythm sleep disorder. Shiftwork sleep disorder is also linked with non 24 hours sleep-wake syndrome [64].

\section{Neurological/psychiatric disorders}

External factors, including jet lag, shift-work, and other environmental factors affect the nervous system that in turn increase the risks associated with psychiatric disorders, which impose serious medical burdens [65-67] and cause disabilities [68]. Continuous light exposure during the night causes circadian misalignment, sleep deprivation and melatonin suppression that result in psychiatric disorder's development such as depression and mood disorders [61].

Bipolar disorder, a severely disabling disorder mainly affects emotional and social behavior. Bright and random light affects perception and thought with the help of genetic and environmental factors [69]. Major depression disorder is yet another nervous system debilitating condition which is symptomized by a loss in work productivity and less interest in social interactions $[70,71]$. Genetic and environmental factors enhance the risk of this neuro-progressive disorder [72].

\section{Cancer}

The disrupted circadian clock through bright light increases the risks of breast cancer $[6,73]$. Irregular light schedules affect clock-controlled genes which are linked with tumor suppressor genes, growth arrest and DNA damage-inducible alpha protein, murine double minute oncogene and P53 encoding genes [6,74]. Per1 and per2 are tumor repressors [75], whereas in human mammary cells PER2 induces estradiol (E2) either by reducing estrogen receptor a response to E2 or enhancing E2 activation. Mutated PER2 increases the cancer development rate by altering the normal functions of CMYC, CYCLIN D1, CYCLIN A, MDM-2, and GADD45A and P53 [76,77]. Non 24 hours rotation of light schedules, specifically exposure to bright light at night, accelerate the rate of colonic adenoma, colonic cancer, and breast cancer by affecting the normal expression of PER1, PER2 and PER3 [78,79]. Disrupted circadian system inactivates or suppresses P53 and overexpresses MYC [76,80] which induces tumor-genesis [6]. Long-term exposure to bright light at night affects IFNY (interferon gamma), Aln repetitive elements and suppresses melatonin [81]. It further impacts the immune system to develop malignancies [6].

\section{Conclusions and benchmark prospects}

Clock genes play a crucial role in maintenance of behavior and other body functions. Avoiding exposure to irregular dark-light cycles and environmental stresses is nearly impossible in today's world. These external factors have strong impacts on internal 
clock system, which make it necessary to be aware of complications related to them. Focusing on precautionary measurements is important to eliminate the chances of related health-risking disorders such as cancer, cardiac system disorders, sleep related disorders, depressive and other psychiatric disorders. Investigations related to changes in environmental conditions and associated disorders are needed to be carried which must focus on the genetic level alterations. Only in this way proper cure or precautions will become possible in future.

\section{Acknowledgement}

Scientific research and service platform fund of Henan Province (2016151); The fund of scientific and technological innovation team of water ecological security for Water Source Region of Mid-line of South-to-North Diversion Project of Henan Province (17454).

Sponsored by CAS-TWAS president's fellowship for international students.

The authors are thankful to Kayla Riley for providing editing and proofreading services.

\section{Competing Interests}

The authors have declared that no competing interest exists.

\section{References}

1. West AC, Smith L, Ray DW, et al. Misalignment with the external light environment drives metabolic and cardiac dysfunction. Nat Commun. 2017;8(1):1-10.

2. Woelders T, Beersma DGM, Gordijn MCM, et al. Daily Light Exposure Patterns Reveal Phase and Period of the Human Circadian Clock. J Biol Rhythms. 2017;32(3):274-86.

3. Keller LK, Grünewald B, Vetter C, et al. Not later, but longer: sleep, chronotype and light exposure in adolescents with remitted depression compared to healthy controls. Eur Child Adolesc Psychiatry. 2017;26(10):1233-44.

4. Liyanarachchi K, Ross R, Debono M. Human studies on hypothalamo-pituitary-adrenal (HPA) axis. Best Pract Res Clin Endocrinol Metab. 2017;31(5):459-73.

5. Iggena D, Winter Y, Steiner B. Melatonin restores hippocampal neural precursor cell proliferation and prevents cognitive deficits induced by jet lag simulation in adult mice. J Pineal Res. 2017;62(4):1-12.

6. Haus EL, Smolensky MH. Shift work and cancer risk: potential mechanistic roles of circadian disruption, light at night, and sleep deprivation. Sleep Med Rev. 2013;17(4):273-84

7. Salgado-Delgado R, Angeles-Castellanos M, Saderi N, et al. Food intake during the normal activity phase prevents obesity and circadian desynchrony in a rat model of night work. Endocrinology. 2010;151(3):1019-29.

8. Saksvik IB, Bjorvatn $B$, Hetland $\mathrm{H}$, et al. Individual differences in tolerance to shift work-a systematic review. Sleep Med Rev. 2011;15(4):221-35.

9. Swanson CM, Kohrt WM, Buxton OM, et al. The Importance of the Circadian System \& Sleep for Bone Health. Metabolism; in press.

10. Khan S, Duan P, Yao L, et al. Shift-work mediated disruptions of Circadian rhythms and sleep homeostasis cause serious health problems. Int J Genomics. 2018;2018:1-11.

11. Flo E, Pallesen S, Magerøy N, et al. Shift work disorder in nurses-assessment, prevalence and related health problems. PLoS One. 2012;7(4):e33981.

12. Knutsson A, Bøggild H. Gastrointestinal disorders among shift workers. Scand J Work Environ Health. 2010; 36(2):85-95.

13. Puttonen $S$, Oksanen $T$, Vahtera J. Is shift work a risk factor for rheumatoid. Ann Rheum Dis. 2010;69(4):779-80.

14. Eldevik MF, Flo E, Moen BE, et al. Insomnia, excessive sleepiness, excessive fatigue, anxiety, depression and shift work disorder in nurses having less than 11 hours in-between shifts. PLoS One. 2013;8(8):e70882.
15. Barclay JL, Husse J, Bode B, et al. Circadian desynchrony promotes metabolic disruption in a mouse model of shiftwork. PLoS One. 2012;7(5):e37150.

16. Vadnie CA, McClung CA. Circadian Rhythm Disturbances in Mood Disorders: Insights into the Role of the Suprachiasmatic Nucleus. Neural Plast. 2017;2017:1-28.

17. Albrecht U, Ripperger JA. Clock Genes. Encycl Neurosci. 2009;1:759-62.

18. Takahashi JS. Molecular components of the circadian clock in mammals. Diabetes, Obes Metab. 2015;17(Suppl 1):S6-S11.

19. Ko $\mathrm{CH}$, Takahashi JS. Molecular components of the mammalian circadian clock. Hum Mol Genet. 2006;15(Suppl 2):S271-S277.

20. Zhang L, Chung BY, Lear BC, et al. DN1 p circadian neurons coordinate acute light and PDF inputs to produce robust daily behavior in Drosophila. Curr Biol. 2010;20(7):591-9.

21. Bass J, Takahashi JS. Circadian integration of metabolism and energetics. Science. 2010;330(6009):1349-54

22. Takahashi JS, Hong $\mathrm{H}-\mathrm{K}, \mathrm{Ko} \mathrm{CH}$, et al. The genetics of mammalian circadian order and disorder: implications for physiology and disease. Nat Rev Genet. 2008;9(10):764-75.

23. Lamia KA, Storch K-F, Weitz CJ. Physiological significance of a peripheral tissue circadian clock. Proc Natl Acad Sci. 2008;105(39):15172-7.

24. Turek FW, Joshu C, Kohsaka A, et al. Obesity and metabolic syndrome in circadian Clock mutant mice. Science. 2005;308(5724):1043-5.

25. Nakamura W, Yamazaki S, Takasu NN, et al. Differential response of Period 1 expression within the suprachiasmatic nucleus. J Neurosci. 2005;25(23):5481-7.

26. Rijo-Ferreira F, Carvalho T, Afonso C, et al. Sleeping sickness is a circadian disorder. Nat Commun. 2018;9:62

27. Ho JM, Ducich NH, Nguyen N-QK, et al. Acute sleep disruption- and high-fat diet-induced hypothalamic inflammation are not related to glucose tolerance in mice. Neurobiol Sleep Circadian Rhythm; in press.

28. Melanson EL, Ritchie HK, Dear TB, et al. Daytime bright light exposure, metabolism, and individual differences in wake and sleep energy expenditure during circadian entrainment and misalignment. Neurobiol Sleep Circadian Rhythm; in press.

29. Bano M, Chiaromanni F, Corrias M, et al. The influence of environmental factors on sleep quality in hospitalized medical patients. Front Neurol. 2014;5:1-8.

30. Halperin D. Environmental noise and sleep disturbances: A threat to health? Sleep Sci. 2014;7(4):209-12.

31. Quera Salva MA, Hartley S, Léger D, et al. Non-24-Hour Sleep-Wake Rhythm Disorder in the Totally Blind: Diagnosis and Management. Front Neurol. 2017:8:1-7.

32. Sehgal A, Mignot E. Genetics of sleep and sleep disorders. Cell. 2011;146(2):194-207.

33. Musiek ES, Videnovic A. Sleep and clocks implications for brain health. Neurobiol Sleep Circadian Rhythm. 2017;2:1-3

34. Gooley JJ, Mohapatra L, Twan DCK. The role of sleep duration and sleep disordered breathing in gestational diabetes mellitus. Neurobiol Sleep Circadian Rhythm; in press.

35. Winkelmann J, Czamara D, Schormair B, et al. Correction: Genome-Wide Association Study Identifies Novel Restless Legs Syndrome Susceptibility Loci on 2p14 and 16q12.1. PLoS Genet. 2011;7(8): e1002171.

36. Stefansson H, Rye DB, Hicks A, et al. A genetic risk factor for periodic limb movements in sleep. N Engl J Med. 2007;357(7):639-47.

37. Winkelmann J, Lichtner P, Schormair B, et al. Variants in the neuronal nitric oxide synthase (nNOS, NOS1) gene are associated with restless legs syndrome. Mov Disord. 2008;23(3):350-8.

38. Rihel J, Prober DA, Arvanites A, et al. Zebrafish behavioral profiling links drugs to biological targets and rest/wake regulation. Science. 2010:327(5963):348-51.

39. Funato H, Miyoshi C, Fujiyama $T$, et al. Forward-genetics analysis of sleep in randomly mutagenized mice. Nature. 2016;539(7629):378-83.

40. Allebrandt KV, Amin N, Müller-Myhsok B, et al. A KATP channel gene effect on sleep duration: from genome-wide association studies to function in Drosophila. Mol Psychiatry. 2013;18(1):122-32.

41. Markwald RR, Melanson EL, Smith MR, et al. Impact of insufficient sleep on total daily energy expenditure, food intake, and weight gain. Proc Natl Acad Sci. 2013;110(14): 5695-700.

42. Depner CM, Stothard ER, Wright Jr KP. Metabolic consequences of sleep and circadian disorders. Curr Diab Rep. 2014;14(7):1-9.

43. Iwamoto A, Kawai M, Furuse M, et al. Effects of chronic jet lag on the central and peripheral circadian clocks in $\mathrm{CBA} / \mathrm{N}$ mice. Chronobiol Int. 2014;31(2):189-98.

44. Drake CL, Wright KP. Shift work, shift-work disorder, and jet lag. Princ Pract sleep Med. 2011;1:784-98.

45. Ruggiero JS, Redeker NS. Effects of napping on sleepiness and sleep-related performance deficits in night-shift workers: a systematic review. Biol Res Nurs. 2014;16(2):134-42.

46. Carpen JD, Archer SN, Skene DJ, et al. A single-nucleotide polymorphism in the 5 '-untranslated region of the hPER2 gene is associated with diurnal preference. J Sleep Res. 2005;14(3):293-7.

47. Mishima K, Tozawa T, Satoh K, et al. The 3111T/C polymorphism of hClock is associated with evening preference and delayed sleep timing in a Japanese population sample. Am J Med Genet Part B Neuropsychiatr Genet. 2005;133(1):101-4. 
48. Pereira DS, Tufik S, Louzada FM, et al. Association of the length polymorphism in the human Per3 gene with the delayed sleep-phase syndrome: does latitude have an influence upon it. Sleep. 2005;28(1):29-32.

49. Tafti M, Dauvilliers Y, Overeem S. Narcolepsy and familial advanced sleep-phase syndrome: molecular genetics of sleep disorders. Curr Opin Genet Dev. 2007;17(3):222-7.

50. Shanware NP, Hutchinson JA, Kim SH, et al. Casein kinase 1-dependent phosphorylation of familial advanced sleep phase syndrome-associated residues controls PERIOD 2 stability. J Biol Chem. 2011;286(14):12766-74.

51. Scheer FAJL, Hilton MF, Mantzoros CS, et al. Adverse metabolic and cardiovascular consequences of circadian misalignment. Proc Natl Acad Sci. 2009;106(11):4453-8.

52. Qian J, Frank AJLS. Circadian system and glucose metabolism. Implications for physiology and disease.Trends Endocrinol Metab. 2016;27(5):282-93.

53. Reutrakul S, Van Cauter E. Interactions between sleep, circadian function, and glucose metabolism: Implications for risk and severity of diabetes. Ann N Y Acad Sci. 2014;1311(1):151-73.

54. Oishi K, Ohyama S, Higo-yamamoto S. Biochemical and Biophysical Research Communications Chronic sleep disorder induced by psychophysiological stress induces glucose intolerance without adipose in $\mathrm{fl}$ ammation in mice. Biochem Biophys Res Commun. 2018;495(4):2616-21.

55. Takeda N, Maemura K. Circadian clock and cardiovascular disease. J Cardiol. 2011;57(3):249-56.

56. Rahman SA, Marcu S, Kayumov L, et al. Altered sleep architecture and higher incidence of subsyndromal depression in low endogenous melatonin secretors. Eur Arch Psychiatry Clin Neurosci. 2010;260(4):327-35.

57. Pandi-Perumal SR, Srinivasan V, Spence DW, et al. Role of the melatonin system in the control of sleep. CNS Drugs. 2007;21(12):995-1018.

58. Vogel M, Braungardt T, Meyer W, et al. The effects of shift work on physical and mental health. J Neural Transm. 2012;119(10):1121-32.

59. Di ML, Waage S, Pallesen S, et al. Shift work disorder in a random population sample-prevalence and comorbidities. PLoS One. 2013;8(1):e55306.

60. Czeisler CA, Walsh JK, Roth T, et al. Modafinil for excessive sleepiness associated with shift-work sleep disorder. N Engl J Med. 2005;353(5):476-86.

61. Smith MR, Eastman CI. Shift work: health, performance and safety problems, traditional countermeasures, and innovative management strategies to reduce circadian misalignment. Nat Sci Sleep. 2012;4:111-32.

62. Markwald RR, Wright Jr KP. Circadian misalignment and sleep disruption in shift work: Implications for fatigue and risk of weight gain and obesity. In: Shiromani P, Horvath T, Redline S, et al, ed. Sleep Loss and Obesity. New York: Springer; 2012: 101-18.

63. Buxton OM, Cain SW, O'Connor SP, et al. Adverse metabolic consequences in humans of prolonged sleep restriction combined with circadian disruption. Sci Transl Med. 2012;4(129):129-43.

64. Sack RL, Auckley D, Auger RR, et al. Circadian rhythm sleep disorders: part I, basic principles, shift work and jet lag disorders. Sleep. 2007;30(11):1460-83.

65. Hawi Z, Cummins TDR, Tong J, et al. The molecular genetic architecture of attention deficit hyperactivity disorder. Mol Psychiatry. 2015;20(3):289-97.

66. Jeste SS, Geschwind DH. Disentangling the heterogeneity of autism spectrum disorder through genetic findings. Nat Rev Neurol. 2014;10(2):74-81.

67. Milham MP. Open neuroscience solutions for the connectome-wide association era. Neuron. 2012;73(2):214-8.

68. Zhao Y, Castellanos FX. Annual Research Review: Discovery science strategies in studies of the pathophysiology of child and adolescent psychiatric disorders-promises and limitations. J Child Psychol Psychiatry. 2016;57(3):421-39.

69. Kieseppä T, Partonen T, Haukka J, et al. High concordance of bipolar I disorder in a nationwide sample of twins. Am J Psychiatry. 2004;161(10):1814-21.

70. Kessler RC, Chiu WT, Demler O, et al. Prevalence, severity, and comorbidity of 12-month DSM-IV disorders in the National Comorbidity Survey Replication. Arch Gen Psychiatry. 2005;62(6):617-27.

71. Koido K, Traks T, Balõtšev R, et al. Associations between LSAMP gene polymorphisms and major depressive disorder and panic disorder. Transl Psychiatry. 2012;2(8):e152.

72. Moylan S, Maes M, Wray NR, et al. The neuroprogressive nature of major depressive disorder: pathways to disease evolution and resistance, and therapeutic implications. Mol Psychiatry. 2013;18(5):595-606.

73. Filipski E, Innominato PF, Wu M, et al. Effects of light and food schedules on liver and tumor molecular clocks in mice. J Natl Cancer Inst. 2005;97(7):507-17.

74. Khan S, Ullah MW, Siddique R, et al. Role of Recombinant DNA Technology to Improve Life. Int J Genomics. 2016;2016.

75. Xiang S, Coffelt SB, Mao L, et al. Period-2: a tumor suppressor gene in breast cancer. J Circadian Rhythms. 2008;6(1):1-4.

76. Yang X, Wood PA, Oh EY, et al. Down regulation of circadian clock gene Period 2 accelerates breast cancer growth by altering its daily growth rhythm. Breast Cancer Res Treat. 2009;117(2):423-31.

77. Gery S, Komatsu N, Baldjyan L, et al. The circadian gene per1 plays an important role in cell growth and DNA damage control in human cancer cells. Mol Cell. 2006;22(3):375-82

78. Wood PA, Yang X, Taber A, et al. Period 2 mutation accelerates ApcMin/+ tumorigenesis. Mol Cancer Res. 2008;6(11):1786-93.

79. Davis S, Mirick DK. Circadian disruption, shift work and the risk of cancer: a summary of the evidence and studies in Seattle. Cancer causes Control. 2006;17(4):539-45.
80. Lee S, Donehower LA, Herron AJ, et al Disrupting circadian homeostasis of sympathetic signaling promotes tumor development in mice. PLoS One. 2010;5(6):e10995.

81. Zhu Y, Stevens RG, Hoffman AE, et al. Epigenetic impact of long-term shiftwork: pilot evidence from circadian genes and whole-genome methylation analysis. Chronobiol Int. 2011;28(10):852-61. 